\title{
Nonrepetitive colorings of graphs
}

\author{
Noga Alon ${ }^{1}$ and Jarosław Grytczuk ${ }^{2}$ \\ ${ }^{1}$ Schools of Mathematics and Computer Science, Raymond and Beverly Sacler Faculty of Exact Sciences, Tel Aviv \\ University, Tel Aviv 69978, Israel, nogaa@tau.ac.il \\ ${ }^{2}$ Faculty of Mathematics, Computer Science and Econometrics, University of Zielona Góra, 65-516 Zielona Góra, \\ Poland,J.Grytczuk@wmie.uz.zgora.pl
}

\begin{abstract}
A vertex coloring of a graph $G$ is $k$-nonrepetitive if one cannot find a periodic sequence with $k$ blocks on any simple path of $G$. The minimum number of colors needed for such coloring is denoted by $\pi_{k}(G)$. This idea combines graph colorings with Thue sequences introduced at the beginning of 20th century. In particular Thue proved that if $G$ is a simple path of any length greater than 4 then $\pi_{2}(G)=3$ and $\pi_{3}(G)=2$. We investigate $\pi_{k}(G)$ for other classes of graphs. Particularly interesting open problem is to decide if there is, possibly huge, $k$ such that $\pi_{k}(G)$ is bounded for planar graphs.
\end{abstract}

Let $k \geq 2$ be a fixed integer. A coloring $f$ of the vertices of a graph $G$ is $k$-repetitive if there is $n \geq 1$ and a simple path $v_{1} v_{2} \ldots v_{k n}$ of $G$ such that $f\left(v_{i}\right)=f\left(v_{j}\right)$ whenever $i-j$ is divisible by $n$. Otherwise $f$ is called $k$-nonrepetitive. The minimum number of colors needed for a $k$-nonrepetitive coloring of $G$ is denoted by $\pi_{k}(G)$. Notice that any 2 -nonrepetitive coloring must be proper in the usual sense, while this is not necessarily the case for $k \geq 3$.

By the 1906 theorem of Thue [6] $\pi_{2}(G) \leq 3$ and $\pi_{3}(G) \leq 2$ if $G$ is a simple path of any length. Let $\pi_{k}(d)$ denote the supremum of $\pi_{k}(G)$, where $G$ ranges over all graphs with $\Delta(G) \leq d$. A simple extension of probabilistic arguments from [2] (for $k=2$ ) shows that there are absolute positive constants $c_{1}$ and $c_{2}$ such that

$$
c_{1} \frac{d^{k /(k-1)}}{(\log d)^{1 /(k-1)}} \leq \pi_{k}(d) \leq c_{2} d^{k /(k-1)} .
$$

Moreover, one can show that for each $d$ there exists a sufficiently large $k=k(d)$ such that $\pi_{k}(d) \leq d+1$. On the other hand, any $\lfloor d / 2\rfloor$-coloring of a $d$-regular graph of girth at least $2 k+1$ is $k$-repetitive. The maximum number $t(d)$ such that for each $k$ there is a $d$-regular graph $G$ with $\pi_{k}(G)>t(d)$ is not known for $d \geq 3$.

Kündgen and Pelsmajer [4] and Barát and Varjú [3] proved independently that $\pi_{2}(G)$ is bounded for graphs of bounded treewidth. By the result of Robertson and Seymour [5] it follows that if $H$ is any fixed planar graph then $\pi_{k}(G)$ is bounded for graphs not containing $H$ as a minor. However, it is still not known whether there are some constants $k$ and $c$ such that $\pi_{k}(G) \leq c$ for any planar graph $G$. The least possible constant $c$ for which this could hold (with possibly huge $k$ ) is $c=4$.

In a weaker version of the problem we ask for nonrepetitive colorings of subdivided graphs. By the result of Thue every graph has a (sufficiently large) subdivision which is nonrepetitively 5-colorable (for any $k \geq 2$ ). Clearly this cannot happen for all graphs if we restrict the number of vertices added to an 1365-8050 @ 2005 Discrete Mathematics and Theoretical Computer Science (DMTCS), Nancy, France 
edge. For instance, any $c$-coloring of the complete graph $K_{n}$, with each edge subdivided by at most $r$ vertices, is 2-repetitive if $c<\log _{r} \log _{2}(n / r)$. The question if there are constants $c, k$, and $r$ such that each planar graph $G$ has an $r$-restricted subdivision $S$ with $\pi_{k}(S) \leq c$, is open.

There are many interesting connections of this area to other graph coloring topics. Let $s(G)$ be the star chromatic number of a graph $G$, that is, the least number of colors in a proper coloring of the vertices of $G$, with additional property that every two color classes induce a star forest. It is not hard to see that $\pi_{2}(G) \geq s(G)$ for any graph $G$. Hence, by the results of Albertson et al. [1] it follows that there are planar graphs with $\pi_{2}(G) \geq 10$, and for each $t$ there are graphs of treewidth $t$ with $\pi_{2}(G) \geq\left(\begin{array}{c}t+1 \\ 2\end{array}\right)$.

\section{References}

[1] M. O. Albertson, G. G. Chappell, H. A. Kierstead, A. Kündgen, R. Ramamurthi, Coloring with no 2-colored $P_{4}$ 's, Electron. J. Combinat., 11 (2004) R\#26.

[2] N. Alon, J. Grytczuk, M. Hałuszczak, O. Riordan, Nonrepetitive colorings of graphs, Random Struct. Alg. 21 (2002), 336-346.

[3] J. Barát, P. P. Varjú, Some results on square-free colorings of graphs, manuscript.

[4] A. Kündgen, M.J. Pelsmajer, Nonrepetitive colorings of graphs of bounded treewidth, manuscript.

[5] N. Robertson, P.D. Seymour, Graph minors V: Excluding a planar graph, J. Combin. Theory Ser. B 41 (1986), 92-114.

[6] A. Thue, Über unendliche Zeichenreichen, Norske Vid Selsk. Skr. I. Mat. Nat. Kl. Christiana, (1906), $1-67$. 